\title{
Properties of SS433 and ultraluminous X-ray sources in external galaxies
}

\author{
S. Fabrika, S. Karpov, P. Abolmasov and O. Sholukhova \\ Special Astrophysical Observatory of the Russian AS, Nizhnij Arkhyz 369167, Russia \\ email: fabrika@sao.ru,karpov@sao.ru,pasha@sao.ru,olga@sao.ru
}

\begin{abstract}
We suggest that the ultraluminous X-ray sources located in external galaxies (ULXs) are supercritical accretion disks like that in SS433, observed close to the disk axis. We estimate parameters of the SS433 funnel, where the relativistic jets are formed. Emergent X-ray spectrum in the proposed model of the multicolor funnel (MCF) is calculated. The spectrum can be compared with those of ULXs. We predict a complex absorption-line spectrum with broad and shallow $\mathrm{K} \alpha / \mathrm{Kc}$ blends of the most abundant heavy elements and particular temporal variability. Another critical idea comes from observations of nebulae around the ULXs. We present results of 3D-spectroscopy of nebulae of two ULXs located in Holmberg II and NGC6946. In both cases the nebula is found to be powered by the central black hole. The nebulae are compared with SS433 nebula (W50).
\end{abstract}

Keywords. X-ray sources, accretion disks, jets, individual: SS433.

\section{Introduction}

The main properties of the ultraluminous X-ray sources (ULXs) - huge luminosities $\left(10^{39-41} \mathrm{erg} / \mathrm{s}\right)$, diversity of X-ray spectra, strong variability, connection with starforming regions, their surrounding nebulae, were reviewed by Ward (2005). ULXs may be supercritical accretion disks observed close to the disk axis in close binaries with a stellar mass black hole or microquasars (Fabrika \& Mescheryakov (2001); King et al. (2001); Koerding et al. (2001)), or they may be intermediate-mass black holes with "normal" accretion disks (Colbert \& Mushotzky (1999); Miller, Fabian \& Miller (2004)). It is also possible that ULXs are not homogeneous class of objects. It was suggested originally by Katz (1987) that SS433 being observed close to the jet axis will be extremely bright Xray source. Fabrika \& Mescheryakov (2001) discussed observational properties of face-on SS433-like objects and concluded that they may appear as a new type of extragalactic $\mathrm{X}$-ray sources. Here we continue to develop this idea.

\section{The funnel in SS433 disk}

The main difference between SS433 and other known X-ray binaries is highly supercritical and persistent mass accretion rate $\left(\sim 10^{-4} M_{\odot} / y\right)$ onto the relativistic star (a probable black hole, $\left.\sim 10 M_{\odot}\right)$, which has led to the formation of a supercritical accretion disk and the relativistic jets. SS433 properties were reviewed recently by Fabrika (2004).

SS433 is an edge-on system, its total luminosity (mainly in UV) is $L_{b o l} \sim 10^{40} \mathrm{erg} / \mathrm{s}$. A temperature of the source is $T=(5-7) \cdot 10^{4} \mathrm{~K}$, a mass loss rate in the wind is $\dot{M}_{w} \sim 10^{-4} M_{\odot} / y$. Both optical and X-ray jets are well collimated $\left(\sim 1^{\circ}\right)$. SS433 X-ray luminosity (the cooling X-ray jets) is $\sim 10^{4}$ times less than bolometric luminosity, however kinetic luminosity of the jets is very high, $L_{k} \sim 10^{39} \mathrm{erg} / \mathrm{s}$. 
The jets have to be formed in a funnel in the disk and the disk wind. Supercritical accretion disks simulations (Eggum, Coroniti, Katz (1985); Okuda (2002); Okuda et al. (2005)) show that a wide funnel is formed (a full opening angle $\theta_{f} \approx 40^{\circ}-50^{\circ}$ ) close to the black hole. Convection is important in the inner accretion disk outside the funnel walls.

If one adopts for the funnel luminosity, that it is about the same as SS433 bolometric luminosity, $L_{f} \approx L_{b o l}$, (Fabrika \& Mescheryakov (2001); Fabrika \& Karpov (2005)) and the funnel opening angle $\theta_{f} \approx 40^{\circ}-50^{\circ}$, one obtains "observed" face-on luminosity of SS433 $L_{x} \sim 10^{41} \mathrm{erg} / \mathrm{s}$ and expected frequency of such objects $\sim 0.1$ per a galaxy like Milky Way. On the other hand a critical luminosity for a $10 M_{\odot}$ black hole is $L_{e d d} \sim$ $10^{39} \mathrm{erg} / \mathrm{s}$. At highly supercritical accretion rate $\dot{M} / \dot{M}_{c r} \sim 10^{3}$ it increases (the inner disk geometry) by a factor of $\left(1+\ln \left(\dot{M} / \dot{M}_{c r}\right)\right) \sim 10$. A doppler boosting factor is not large for $\operatorname{SS} 433\left(\beta=V_{j} / c=0.26\right)$, it is $1 /(1-\beta)^{2+\alpha} \sim 2.5$. The third factor is the geometrical funneling $\left(\theta_{f} \approx 40^{\circ}-50^{\circ}\right)$, it is $\Omega_{f} / 2 \pi \sim 10$. Thus, one may expect a face-on luminosity of such supercritical disk $L_{e d d} \sim 2 \cdot 10^{41} \mathrm{erg} / \mathrm{s}$.

\section{The multicolor funnel model}

A size of SS433 wind photosphere is $r_{p h \text {,wind }} \sim 10^{12} \mathrm{~cm}$. An estimate of the jet photosphere (Fabrika \& Karpov (2005)), which is a bottom of the wide funnel, gives $r_{p h, j e t} \sim 4 \cdot 10^{9} \mathrm{~cm}$. We find that the jet is not transparent down to the black hole horizon. However, the gas supply to the funnel is very variable due to convection (Okuda et al. (2005)).

Fabrika \& Karpov (2005) developed a simple model of the funnel (multicolor funnel, $\mathrm{MCF}$ ) to estimate the emerging X-ray spectrum. They considered both gas pressure dominated, $T(r) \propto r^{-1}$, and radiation pressure dominated, $T(r) \propto r^{-1 / 2}$ cases. They based on the observed temperature of the wind photosphere in SS433 and found temperatures of the inner funnel walls (at a level of $r_{p h, j e t}$ ), $T_{p h \text {,wind }}$ between $\sim 1.7 \cdot 10^{7} \mathrm{~K}$ and $\sim 1 \cdot 10^{6} \mathrm{~K}$. Below $r_{p h, j e t}$ the walls can not be observed.

In Fig. 1 we present MCF energy spectra with different ratios of radiation to gas pressure $\left(\xi=a T_{0}^{3} / 3 k_{b} n_{0}\right)$ at the deepest visible parts of the funnel's walls $\left(r_{p h, j e t}\right)$. The temperature $T_{0}=1 \mathrm{keV}$ was adopted. They are spectra with no radiation losses in the funnel $\left(\theta_{f} \approx 0\right)$, and with radiation losses included $\left(\theta_{f} \approx 40^{\circ}\right)$. The multicolor disk (MCD) spectrum with the same inner temperature $T_{i n n}=1 \mathrm{keV}$ is shown for comparison. A low-temperature break in the MCF spectra appears due to finite length of the funnel.

The jets velocity $(0.26 c)$ in SS433 and its surprising stability suggest line-locking mechanism (Shapiro, Milgrom \& Rees (1986)) to operate in the funnel. A photosphere of the funnel's inner walls may radiate absorption spectrum with Lc and Kc edges of Hand He-like ions. The MCF model predicts very complex absorption-line spectrum. Fig. 1 presents expected absorption line profiles of OVIII L $\alpha$ and Lc transitions.

The absorption bands should belong to $\mathrm{H}$ - and He-like ions of the most abundant heavy elements and should extend from the Kc to the $\mathrm{K} \alpha$ energies of the corresponding ions. Variations of the gas parameters along the funnel - its velocity, density, temperature and volume filling factor (the collimation) - could make the absorption-line profiles appreciably more complex, necessitating the use of X-ray spectra with high signal/noise ratios in searches for these lines.

The supposition that ULXs are face-on SS433 objects leads to the following predictions: 1) We expect typical MCD-like spectra, however the MCF spectra may be more diverse depending on the funnel parameters. 2) Absorption spectrum with broad and shallow $\mathrm{L} \alpha / \mathrm{Lc}, \mathrm{K} \alpha / \mathrm{Kc}$ blends of the most abundant heavy elements. 3) Temporal variability on 

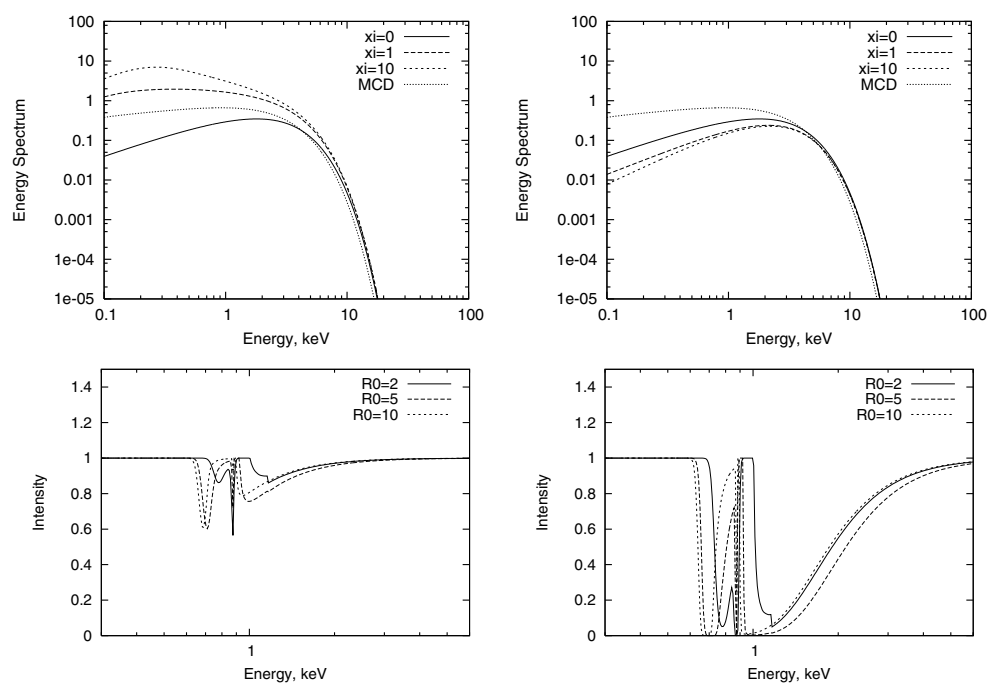

Figure 1. Top: energy spectra of the multicolor funnel (MCF) compared with the MCD spectrum with the same inner temperature $1 \mathrm{keV}$. Bottom: absorption line profiles of OVIII L $\alpha$ and Lc transitions at different efficiencies of jet acceleration for optically thin (left) and for optically thick (right) regimes. The acceleration starts at $R_{0}=1$ (in units of the jet bottom photosphere, $\left.r_{p h, j e t}\right)$, and ends at $R_{0}=2,5,10$. The line intensities are in arbitrary scale.

time scales $r_{p h, w i n d} / c \sim 30 \mathrm{sec}$ and $r_{p h, j e t} / c \sim 0.1$ sec. 4$)$ A typical accretion disk power density spectrum at scales $>0.1 \mathrm{sec} .5)$ Very bright UV source $\left(L_{U V} \sim 10^{40} \mathrm{erg} / \mathrm{s}\right.$, $\left.T \geqslant 10^{5} \mathrm{~K}\right)$ is less collimated than X-ray source. The predicted complexity of the dependence of the absorption-line profiles on the structure of the funnel and mechanisms of acceleration and collimation of the gas in the funnel presents excellent opportunities for direct probing of these structures in supercritical accretion disks and for studies of mechanisms of jet formation.

\section{W50 and ULXs nebulae}

The radio nebula W50 was produced (or distorted) by SS433 jets. We show W50 in Fig. 2 together with nebulae surrounding two ULXs in Holmberg II and NGC6946 galaxies, which were studied recently by Integral-field spectroscopy method (Lehmann et al. (2005); Fabrika, Abolmasov \& Sholukhova (2005)). W50 contains bipolar nebula with optical filaments located at $\pm 0.5^{\circ}$ or $\pm 50 p c$ from SS433 at places of jets termination. A total energy of the nebula is $E_{k} \sim 2 \cdot 10^{51} \mathrm{erg}$ (Zealey et al. (1980)), which corresponds to the jet kinetic luminosity $L_{k} \sim 3 \cdot 10^{39} \mathrm{erg} / \mathrm{s}$ for 20000 years. The observed velocity dispersion in the filaments is $\sim 50 \mathrm{~km} / \mathrm{s}$, however [NII] $/ \mathrm{H} \alpha$ line ratio corresponds to dispersion $\sim 300 \mathrm{~km} / \mathrm{s}$. SS433 is an edge-on system, $i=79^{\circ}$. If one takes into account this factor, the velocity dispersion may reach $250-300 \mathrm{~km} / \mathrm{s}$.

ULXs are located frequently in bubble-like nebulae. New data (Pakull (2005)) show that the nebulae are expanding with a velocity $\sim 80 \mathrm{~km} / \mathrm{s}$ (up to $\sim 250 \mathrm{~km} / \mathrm{s}$ ). In two well-studied ULXs nebulae radial velocity gradients in a high ionisation emission line He II $\lambda 4686$ were found. They are $\pm 50 \mathrm{~km} / \mathrm{s}$ on spatial scale $\sim \pm 30 p c$ in Holmberg II ULX-1 (Lehmann et al. (2005)), and $\sim \pm 50 \mathrm{~km} / \mathrm{s}$ on a scale $\pm 20 p c$ in NGC6946 ULX1 (Fabrika, Abolmasov \& Sholukhova (2005)). In all cases line ratios indicate shock ionisation. This means that the nebulae are powered by the central source, probably via jets activity. 

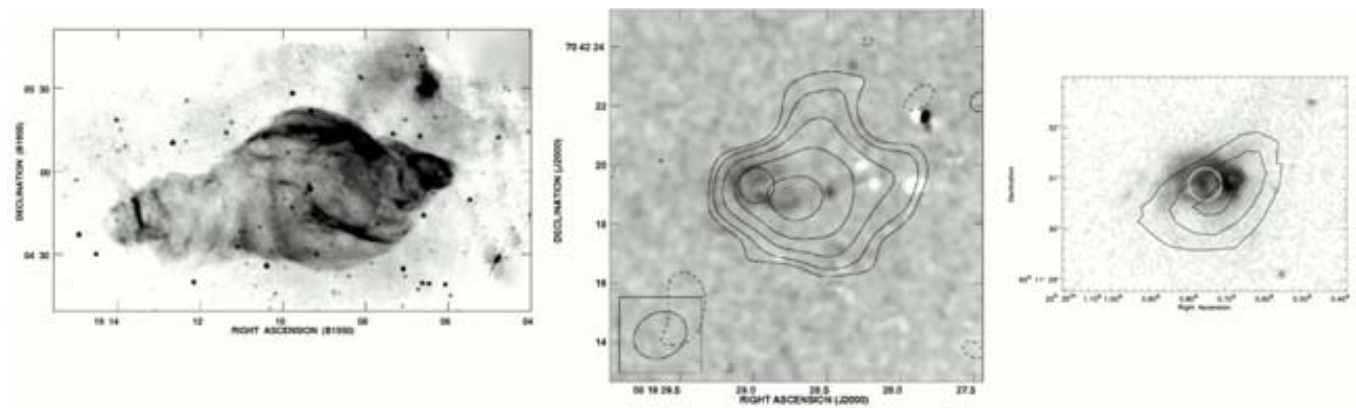

Figure 2. Three nebulae on the same scale in parsecs. VLA image of W50 (Dubner et al. (1998)) with SS433 in center, left; Holmberg II ULX-1 in HST HeII image with VLA isophotes, middle (Miller, Mushotzky \& Neff (2005)) and NGC6946 ULX-1 in HST H $\alpha+$ [SII] image with VLA isophotes, right (van Dyk et al. (1994)). Circles show X-ray Chandra positions.

The nebulae in Holmberg II and NGC6946 have circle-like features in the line-images. In both cases the radio sources are shifted to a brighter circle-like feature and in the both cases this part of the nebulae is approaching, the opposite part is receding (Lehmann et al. (2005); Fabrika, Abolmasov \& Sholukhova (2005)). At some imagination one may conclude that the nebulae around these two ULXs are face-on versions $\left(i=10^{\circ}-30^{\circ}\right)$ of the SS433 nebula.

\section{Acknowledgements}

The work is supported by RFBR under grants number 03-02-16341 and 04-02-16349.

\section{References}

Colbert, E.J.M. \& Mushotzky, R.F. 1999, ApJ 51989

Dubner, G.M., Holdaway, M., Goss, W.M., \& Mirabel, I.F. 1998, AJ 1161842

Eggum, G.E., Coroniti, F.V., \& Katz, J.I. 1985. ApJ (Letters) 298 L41

Fabrika, S. 2004, Astrophysics and Space Physics Reviews, vol. 12, p. 1

Fabrika, S. \& Mescheryakov, A. 2001, in: R.T. Schilizzi (ed.), Galaxies and their Constituents at the Highest Angular Resolution, IAU Symp. N205 (Manchester, UK), p. 268

Fabrika, S. \& Karpov, S. 2005, in preparation

Fabrika, S., Abolmasov, P., \& Sholukhova, O. 2005, in preparation

Katz, J.J. 1987, ApJ 317264

King, A.R., Davies, M.B., Ward, M.J., Fabbiano, G., \& Elvis, M. 2001, ApJ (Letters) 552 L109

Koerding, E., Falcke, H., Markoff, S., \& Fender, R. 2001, Astron. Gesells. Meet. Abstr. 18176

Lehmann, I., Becker, T., Fabrika, S., Roth, M., Miyaji, T., Afanasiev, V., Sholukhova, O., Sanchez, S.F., Greiner, J., Hasinger, G., Costantini, E., Surkov, A., \& Burenkov, A. 2005, A\&A 431847

Miller, J.M., Fabian, A.C., \& Miller, M.C. 2004, ApJ (Letters) 614 L117

Miller, N.A., Mushotzky, R.F., \& Neff, S.G. 2005, ApJ (Letters) 623 L109

Okuda, T. 2002, PASJ 54253

Okuda, T., Teresi, V., Toscano, E., \& Molteni, D. 2005, MNRAS 357295

Pakull, M., 2005, this conference

Shapiro, P.R., Milgrom, M., \& Rees, M.J. 1986, ApJSS 60393

van Dyk, S.D., Sramek, R.A., Weiler, K.W., Hyman, S.D., \& Virden, R.E., 1994 ApJ (Letters) $425 \mathrm{~L} 77$

Ward, M.J., 2005, this conference

Zealey, W.J., Dopita, M.A., \& Malin, D.F. 1980, MNRAS 192731 